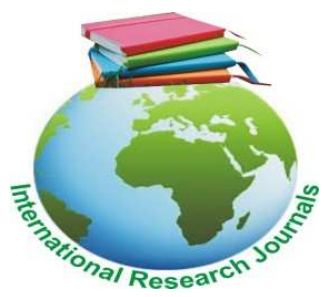

Educational Research (ISSN: 2141-5161) Vol. 8(4) pp. 039-048, October, 2017

Available online@ http://www.interesjournals.org/ER

DOI: http:/dx.doi.org/10.14303/er.2017.216

Copyright (C) 2017 International Research Journals

Review

\title{
Preconception care: a strategy to reduce neonatal/child and maternal mortality
}

\author{
Adewole Abimbola D and Odutayo Patience 0 \\ Department of Nursing, College of Medicine, University of Ibadan, Ibadan, Oyo State \\ Authors corresponding email: dokkybammy@yahoo.com
}

\begin{abstract}
High Neonatal/Child and Maternal mortality still remain an issue of great concern in the developing countries. These mortalities are as a result of conditions or diseases that are preventable only if early detection and interventions are done. These interventions that affect the mother and child and the family at large can be achieved through adequate Preconception Care. Preconception care is therefore the interventions that aim to identify and modify biomedical, behavioral and social risks to a woman's health or pregnancy outcome through prevention and management by emphasizing those factors that must be acted on before conception or early in pregnancy to have maximal impact. Its components includes: Health promotion (for example, education and information dissemination), Nutritional planning and supplementation, Screening and vaccination for diseases known to affect maternal health and pregnancy outcomes and Family Planning. However, majority especially, the adolescences which are the group that is most affected are not aware of the preconception care and its importance before entering into relationship that leads to childbearing. This therefore has prompted the review of this topic.
\end{abstract}

Keywords: Preconception Care, Stategy Neonatal/Child Mortality, Maternal Mortali.

\section{INTRODUCTION}

The American College of Obstetricians and Gynecologists recommend that all health encounters during a woman's reproductive years, particularly those that are a part of preconception care, should include counseling on appropriate health behaviors to optimize pregnancy outcomes and prevent maternal and child mortality (World Health Organization, 2015). Preparing for Life is a unique joint venture which will endeavour to prevent some of life's problems before they begin, rather than try to diminish their consequences after they arise (American College of Obstetricians and Gynecologists, 2014). However, Poor outcomes during pregnancy can seriously have impact on the lives of the women, families and communities (American College of Obstetricians and Gynecologists, 2014). Improving pregnancy outcomes is therefore important for improving the health of the individual and the family.

In order to optimize the chance for a healthy pregnancy and baby, a woman's risk factors are related to varieties of health issues (diet, medications, and exposures) are generally examined, allowing for required modifications during preconception care (Anne et al., 2014). This type of care can help prevent some birth defects and some adverse birth outcomes, such as prematurity (Anne et al, 2014). In contrast, genetic factors result in conditions often perceived to be rare, and thus most women without a family history of birth defects or genetic conditions do not consider themselves at risk. Regardless, a full range of genetic issues should always be considered preconceptionally to improve the chance for a healthy birth outcome.

High Perinatal and Maternal mortality still remain an issue of great concern in the developing countries (Walsh, Feifer et al., 2013). Perinatal mortality refers to deaths of the fetus after 22 weeks of gestation or newborn through the first week after birth, which include pregnancy losses occurring after seven completed months of gestation and the deaths within the first seven days of life which could be due to abnormal presentation of the fetus umbilical cord prolapse, or prolonged labor, neonatal infection, diarrhea, malaria, measles and malnutrition which are all preventable (Walsh et al., 
040 Educ. Res.

2013).Maternal mortality is the death of either a pregnant woman or death of a woman within 42 days of delivery, miscarriage, termination or ectopic pregnancy providing the death is associated with pregnancy or its treatment which could be due to Gestational diabetes, Hypertensive disorders of pregnancy, Anaemia, Multiple pregnancy, inadequate use of antenatal care services, Smoking, Substance misuse, Previous pregnancy problems, Preexisting medical conditions, including epilepsy, mental health problems, cardiac problems, essential hypertension (Walsh et al., 2013). However, all these causes of mortality are preventable when there is adequate health information/education and screening before pregnancy. This can be achieved through proper and adequate preconception care that has its' targets all women of reproductive age, during adolescence, before the first pregnancy, and between pregnancies (Ogle, 2012), (Naish and Roberts, 2012).

Preconception care therefore can be defined as the interventions that aim to identify and modify biomedical, behavioral and social risks to a woman's health or pregnancy outcome through prevention and management by emphasizing those factors that must be acted on before conception or early in pregnancy to have maximal impact (Johnson, et al., 2010). Most causes of Neonatal and maternal mortality are as a result of pregnancies that are unplanned (Singh, 2010). This is rampant among adolescent girls and young women that are thrust into motherhood without the adequate knowledge, skills or support needed to cope with the situation. As a result, they could not have the essential health interventions they needed once a woman and her partner decide to have a child. This is because, these adolescences and young women are not given proper and adequate sex education as early as possible in their developmental stages and more so, the practice of preconception care is not common in developing countries because of inadequate resources, widely dispersed population, literacy and financial status and cultural and traditional practices (Nylander and Adeyemi, 2010).

Adequate implementation of information on care before, during and after pregnancy can improve the outcome of delivery and health of the man and woman (Ekwempu, 2010). Preconception care covers the provision of preventive, primitive or curative health and social interventions before conception occurs (preconception), the provision of interventions in the period extending from 3 months before to 3 months after conception occurs (Periconception care) and the provision of interventions between two pregnancies (interconception care) (World Health Organization, 2015).For the fact that preconception care captures the healthcare of both males and females in all reproductive ages, it makes it the most significant strategy to reduce maternal and neonatal/ child mortality. This write up therefore, aimed at reviewing what preconception care is, components of preconception care, its' importance and how it is one of the strategies to reduce perinatal and maternal mortality.

\section{Overview of preconception care}

Preconception care is the provision of biomedical, behavioural and social health interventions to women and couples before conception occurs. In other words, preconception care as "any intervention provided to women and couples of childbearing age, regardless of pregnancy status or desire, before pregnancy, to improve health outcomes for women, newborns and children" (Johnson et al, 2010). The ultimate aim of preconception is to improve maternal and child health status by reducing behaviours, individual and environmental factors that could contribute to poor maternal and child health both in the short and long term for a desirable outcome through adequate and proper sex and health education and screening/investigations to detect health status and to prevent or treat as the case may be (Jack et al., 2016).

Preconception care addresses both unmarried and married people and should begin early before any pregnancy for a better or best outcome as the case may be. However, in the short term of preconception care, pregnancies that are too early, too close and unplanned could be reduced. When proper investigations and screening are carried out on an individual(male or female) as early as possible, the risk of genetic disorders and environmental exposure that contribute to poor outcome of pregnancies are reduced, thereby, reducing maternal and childhood mortality and also, improving maternal and child health outcomes(Liu, 2010).It also contribute to improving the health and well-being of women in other areas of public health, such as nutrition, infertility and sub fertility, mental health, intimate partner and sexual violence, and substance use(Singh, 2010). Nevertheless, in the long term, if preconception care is given, it could contribute to improving the health of babies and children as they grow into adolescence and adulthood (Liu, 2010. This is possible by supporting women to make well-informed and well-considered decisions about their fertility and their health. More so, preconception care contributes to the social and economic development of families and communities by creating awareness of the importance of men's health and men's behaviours on maternal and child health outcomes and by promoting male involvement in women's care (World Health Organiczation, 2015).

Furthermore, preconception Care is all in interventions that are carried out to improve the reproductive period of life of child bearing age group. These interventions are not in one package and could be delivered both in health facilities and in community settings. According to World Health Organization, 2015, this package includes:

- Health promotion (for example, education and information dissemination) 
- Nutritional planning and supplementation

- $\quad$ Screening and vaccination for diseases known to affect maternal health and pregnancy outcomes.

- Family Planning.

The health conditions and risk factors addressed in preconception care must be detectable in clinical practice (that is, there must be a screening or diagnostic test that can detect it). They must also be sufficiently common and severe to warrant screening. Some conditions are so common that all women will be screened for them as part of preconception care (e.g. chickenpox). Others are only common in certain groups of women (for example, young women have a higher risk of chlamydia), and in these cases only women who appear to be at high risk of the condition will be screened. (Jack et al, 2012).

\section{Importance of preconception care}

According to (Jack et al., 2012), Hundreds of thousands of women die each year as a result of pregnancy complications. While such deaths occur most commonly in developing countries, maternal mortality and morbidity remains a significant threat to women's health in developed countries. Poor outcomes during pregnancy can seriously impact on the lives of women, families and communities. Improving pregnancy outcomes is therefore important for improving the health of the whole family. Poor pregnancy outcomes includes: Maternal illness during pregnancy, miscarriage or stillbirth, poor outcomes for newborn babies, such as low birth weight and birth defects and poor childhood development.

\section{Optimizing health before conception}

It is important to be in the best health before becoming pregnant. For example, higher folate (a vitamin important for cell growth) is required during pregnancy. It is to be increased during the very early stages of pregnancy, even before the individual misses her menstrual bleeding (Jack et al., 2012). Inadequate folate can have a severe impact on the developing foetus, and is associated with neural tube defects. Most women will need to increase their folate intake before pregnancy and so preconception advice regarding folate supplementation is required. The American College of Obstetricians and Gynecologists (ACOG), and other experts strongly recommend that all women of childbearing age get $400 \mathrm{mcg}$ of folic acid every day. Meanwhile, only $30 \%$ of women living are aware of the need for folic acid before pregnancy, meaning that $70 \%$ are unlikely to increase their intake unless they receive advice to do so from their medical personnel (Jack et al., 2012). Studies have shown that, parents of disabled children spend three times more raising their child than parents of non-disabled children and disabled children are $80 \%$ more likely to live in poverty than non-disabled children. However, having a child with an intellectual disability may also involve suffering for the parents, family and caregivers, and create an additional burden for the healthcare system (Frey and Files, 2012). Comprehensive examination of reproductive health is supposed to be carried out in order to protect the health of potential mothers through pregnancy, reducing the occurrence of unsuccessful pregnancy outcomes (including birth defects and genetic diseases), and promoting the birth of healthy infants (Frey et al., 2012). The comprehensive examination includes: comprehensive health screening, family history assessment, referral for carrier screening for cystic fibrosis and predictive genetic testing based on family history when appropriate. Risk factors can include a family history of single gene disorders such as muscular dystrophy, cystic fibrosis, sickle cell disease, and Fragile $X$ syndrome, Chromosomal abnormalities, unexplained mental retardation, multiple miscarriages and major birth defects. Any increase above the baseline population risk would depend upon the precise condition in the family, the inheritance pattern, and the degree of relatedness to the woman or her partner. There are some women with known or suspected genetic disorders, causing increased risk for maternal complications during a pregnancy (Liu, 2010). These complications include the development of pulmonary insufficiency in a woman with cystic fibrosis, sickle cell crisis and other vascular complications in a woman with sickle cell disease, hypoglycemic seizures in women with fatty acid oxidation disorders or glycogen storage disease type $\mathrm{I}$ and hyperammonemia and encephalopathy in women with ornithine transcarbamylase deficiency (Liu, 2010). For each of these and many other genetic conditions, inadequate prenatal and postpartum care and monitoring could lead to significant morbidity or even death. Without doubt, women with genetic conditions need to be aware of the added health risk during a future pregnancy, and they should be monitored by appropriate obstetrics or maternal-fetal medicine specialists (De Weerd et al., 2012).

\section{Preconception care interventions and aspects to consider}

Preconception care is a set of intervention that males and females in the reproductive age need in making decisions in their reproductive lives. According to Center for Disease Control on Preconception Care, 2012, the following interventions can guide a Nurse in guiding her Patients or Clients.

\section{a) Behavioral}

\section{Alcohol misuse}

Women who are heavy drinkers (five or more drinks on 
042 Educ. Res.

one occasion on five or more days in the past 30 days) have a higher risk of cardiac and hepatic complications (Floyd et al., 2015). Any amount of alcohol drinking among pregnant women also puts the fetus at risk for fetal alcohol syndrome (FAS) Floyd et al, 2015). Patients should be informed that prenatal alcohol abuse is a preventable cause of birth defects, including mental retardation and neuro developmental defects. It is recommended that women are counseled preconceptionally about these effects.

\section{Domestic violence}

Trauma, either accidental or intentional is the leading cause of death in women of reproductive age. Violence often begins during pregnancy and if it is already present it may escalate and continue through the postpartum period. Screening women at least once in each trimester is recommended whenever bruising, improbable injury, or depressed mood is noted.

\section{Drug abuse}

The use of illicit drugs and alcohol during pregnancy has adverse effects on the neonate, and these children are at risk for altered neuro developmental outcome and poor health status. Detection and treatment of drugs and alcohol are essential precursors to appropriate therapeutic interventions in the preconception period.

\section{Folic acid}

Neural tube defects (NTDs), such as anencephaly and spinal bifida, have multi factorial origins but their etiology often may involve abnormalities in homocysteine metabolism that are potentially remediable by folic acid dietary supplementations. The first occurrence of NTDs may be reduced if women of reproductive age take $0.4 \mathrm{mg}$ of folic acid daily both before conception and during the first trimester of pregnancy. However, due to this, the American College of Obstetricians and Gynecologists (ACOG), and other experts strongly recommend that all women of childbearing age get $400 \mathrm{mcg}$ of folic acid every day, (ACOG, 2013).

\section{Over-the counter medications}

Talk to your patient about her use of over-the-counter medications, herbal products, vitamins, or nutritional supplements. Certain vitamins in excess are harmful. For example, vitamin $A$ in doses greater than 10,000 international units has been shown to cause severe birth defects when taken during pregnancy (Centers for Disease Control and Prevention, 2012).

\section{Prior pregnancy loss}

Preconceptional counseling is recommended in women who experienced a prior pregnancy loss. During the preconception period, investigate the factors that may have contributed to the previous negative outcome and attempt to assuage guilt and help patients resolve any grief from a previous loss. Preconception care provides recommendations to the patients that may reduce the chances of pregnancy loss (Ogle, 2012). Also, inform patients realistically about the likelihood of successful future childbearing (Ogle, 2012).

\section{Psychosocial concerns}

Psychosocial issues are non-biomedical factors that affect mental and physical wellbeing (Klerman, Jack and Coonrod, 2015). Screening for risk factors may help predict a woman's attentiveness to personal health matters, her use of prenatal services, and the health status of her offspring (Klerman et al., 2015). Psychosocial screening should include assessment of risk factors, such as: barriers to care, unstable housing, unintended pregnancy, communication barriers, nutrition, tobacco use, substance use, depression, safety, intimate partner violence, and stress (Klerman et al., 2015).

\section{Smoking}

Health risks associated with smoking during pregnancy include intrauterine growth restriction, placenta previa, and abruption placetae. Additionally, adverse pregnancy outcomes may occur including premature rupture of membranes, low birth weight, and perinatal mortality. Smokers of reproductive age should be counseled about the associated risks of smoking and the negative outcomes associated with pregnancy.

Both cessation of tobacco use and prevention of smoking relapse are key clinical intervention strategies during preconception and pregnancy. A 5-15 minute counseling session performed by appropriately trained health care providers is most effective with pregnant women who smoke fewer than 20 cigarettes per day. This intervention, known as the $5 \mathrm{~A}$ 's, is appropriate for use during routine prenatal office visits and includes the following five steps: Ask, Advise, Assess, Assist, and Arrange.

\section{b) Chronic diseases}

\section{Asthma}

Asthma during pregnancy requires special attention and comprehensive treatment. Alterations of doses for certain 
medications such as corticosteroids may change. Educating patients preconceptionally would be beneficial to the patients' pregnancy outcome. Additionally, environmental factors such as allergens (animal dander, house-dust mites, cockroaches, pollens, and indoor molds), tobacco smoke, and indoor/outdoor pollutants (wood-burning stoves of fireplaces, unvented stoves, perfumes, cleaning agents) could exacerbate asthma attacks and should be discussed during preconception to limit exposures.

\section{Diabetes}

Preconceptional counseling for women with pregestational diabetes mellitus is beneficial and costeffective. Preconceptional counseling should focus on the importance of euglycemic control before pregnancy, as well as the adverse obstetric and maternal outcomes that can result from poorly controlled diabetes. An evaluation for underlying vasculopathy is advisable and, in selected patients, may include a retinal examination by an ophthalmologist, a 24-hour urine collection for protein excretion and creatinine clearance, and electrocardiography. Daily multivitamins containing at least $0.4 \mathrm{mg}$ of folic acid are particularly important in women with diabetes given their increased risk of neural tube defects. Higher doses of folic acid may be beneficial in some cases, especially in the presence of other risk factors for neural tube defects.

\section{Heart disease}

American College of Obstetricians and Gynaecologists, 2013, women of reproductive age living with heart disease should be counseled about the potential risks associated with pregnancy. Hypertension in pregnancy, specifically preeclampsia and transient hypertension of pregnancy, is associated with increased rates of hypertension and coronary heart disease later in life (Boggesss et al., 2013).

\section{Hypothyroidism}

Women should be counseled preconceptionally about treatment during pregnancy. Treatment of hypothyroidism in pregnant women is the same as for non-pregnant women and involves administering levothyroxine at sufficient dosages to normalize thyroid-stimulating hormone (TSH) levels. Levothyroxine therapy should be adjusted at four-week intervals until TSH levels are stable. Pregnancy increases maternal thyroid hormone requirements in women with hypothyroidism diagnosed before pregnancy (American College of Obstetricians and Gynecologists, 2013).

\section{Obesity}

Obesity may be defined as a body mass index (BMI) of $30 \mathrm{~kg} / \mathrm{m} 2$ or greater. Obstetricians or health care providers should provide preconception counseling and education about the possible complications and should encourage obese patients to undertake a weight reduction program before attempting pregnancy. However, modest reductions in weight may have a beneficial effect on perinatal outcome (Castro et al, 2012). According to American College of Obstetricians and Gynecologists, (2015), weight reduction is not advised during pregnancy but counseling concerning appropriate weight gain is advisable. The goal should be towards development of lasting diet and exercise habits which will help the woman sustain a healthy weight throughout her lifetime American College of Obstetricians and Gynecologists, (2015).

\section{Oral health}

Dental care is encouraged as appropriate before and during pregnancy. Some studies have found an association between periodontal disease and poor pregnancy outcomes of premature delivery, low birth weight and preeclampsia. Additional research is needed in this area.

\section{c) Genetics}

\section{Cystic Fibrosis (CF)}

Preconception carrier screening for $\mathrm{CF}$ allows carrier couples to consider all reproductive options. CF screening is recommended for all couples, especially with the individuals with a family history of CF and medical records of the affected family members should be obtained.

\section{Maternal Phenylketonurea (PKU)}

Routine screening for PKU in newborns and early dietary therapy with a phenylalanine-restricted diet have markedly reduced mental retardation in affected individuals. It has been suggested that dietary control should be implemented at least three months prior to conception to help prevent fetal structural defects, cardiac defects, low birth weight, microcephaly, and mental retardation (American College of Obstetricians and Gynecologists, 2010).

\section{Sickle cell anemia}

Pregnancy in women with sickle cell disease is associated 
044 Educ. Res.

with an increased risk of morbidity and mortality because of the combination of underlying hemolytic anemia and multiorgan dysfunction associated with this disorder American College of Obstetricians and Gynaecologists, 2014). Pregnant patients with sickle cell disease need increased prenatal folic acid supplementation. A recommended $400 \mathrm{mcg}$ per day of folic acid should be prescribed to this category of patients due to the continual turnover of red blood cells (American College of Obstetricians and Gynecologists, 2013).

\section{d) Medication}

Medication use should be continued to control disease in women during the preconception period. Switching medication may be appropriate during the preconception period if suitable alternatives exist with less risk to the pregnant woman or fetus. General statements may be made about the teratogenetic potential of prescription drugs, however, maternal condition and treatment needs should be considered, weighing the benefit to the mother with the risk to the fetus. The U.S. Food and Drug Administration has defined five risk categories (A, B, C, $D, X)$ that are used by manufacturers to rate their products for use during pregnancy. Certain drugs taken preconceptionally may be a risk factor for negative pregnancy outcomes. Some examples of drugs which should be managed carefully during the preconception period are:

\section{Isotretinoins}

If used in pregnancy to treat acne, it can result in miscarriage and birth defects. Pregnancy prevention should be practiced in women of reproductive age taking these drugs.

\section{Anti-epileptic drugs}

Certain types of these drugs are teratogens (e.g. valproic acid).

\section{Oral anticoagulants}

Drugs for management of blood clotting such as Warafin have shown to be teratogenic. Early exposure during pregnancy could be avoided preconceptionally by switching drugs.

\section{e) Sexually Transmitted Infections (STIs)}

STIs can have harmful effects on pregnant women, their partners, and their fetuses (Centers of Disease Control and Prevention, 2012). All women of reproductive age and their sex partners should be asked about STIs, counseled about the possibility of perinatal infections during pregnancy, and given access to treatment if needed preconceptionally and during pregnancy (Bourke et al., 2014).

\section{Bacterial Vaginosis (BV)}

Evaluation for BV might be conducted during the first prenatal visit for asymptomatic patients who are at high risk for preterm labor (for example, those who have a history of a previous preterm delivery).

\section{Chlamydia}

All pregnant women should be routinely tested for Chlamydia trachomatis at the first prenatal visit.

\section{Gonorrhea}

All pregnant women at risk for gonorrhea or living in an area in which the prevalence of Neisseria gonorrhoeae is high should be tested at the first prenatal visit forN. gonorrhoeae. A repeat test should be performed during the third trimester for those at continued risk( Bourke et al, 2014).

\section{Hepatitis B}

All pregnant women should be routinely tested for hepatitis B surface antigen ( $\mathrm{HBsAg}$ ) during an early prenatal visit (for example, first trimester) in each pregnancy even if previously vaccinated or tested. All laboratories that conduct HBsAg tests should use an HBsAg test that is FDA-cleared and should perform testing according to the manufacturers labeling, including testing of initially reactive specimens with a licensed neutralizing confirmatory test.

\section{Hepatitis C}

All pregnant women at high risk for hepatitis $C$ infection should be tested for hepatitis $C$ antibodies at the first prenatal visit. Women at high risk include those with a history of injection drug use and those with a history of blood transfusion or organ transplantation before 1992.

\section{Hiv}

All pregnant women in the United States should be tested 
for HIV infection as early in pregnancy as possible.

\section{Cervical cytology}

Cervical cytology testing should be obtained at the first prenatal visit if none has been documented during the preceding year.

\section{Syphilis}

A serologic test should be performed on all pregnant women during the first prenatal visit vaccination.

\section{Human papillomavirus}

The U.S. Food and Drug Administration has approved a quadrivalent human papillomavirus (HPV) vaccine for females aged 9-26 years. ACOG recommends the vaccination of females in this age group. The quadrivalent HPV vaccine has been classified by the FDA as pregnancy category $B$. Thus, vaccination use during pregnancy is not recommended at this time.

\section{Influenza}

Women who will be pregnant during the influenza season should be vaccinated with the influenza vaccine (American College of Obstetricians and Gynecologists, 2014). Any theoretical risk of the vaccination is outweighed by its benefits. Likewise, the benefits of the vaccine outweigh any unproven potential concerns about traces of thimerosal preservative, which exist only in the multidose vials. It should be noted that the intranasal vaccine spray contains a live, attenuated virus and should not be used during pregnancy.

\section{Rubella seronegativity}

The rubella vaccine is a live attenuated virus and is highly effective with few side effects in rubella susceptible women of reproductive age. Rubella vaccination is not recommended during pregnancy and women should be advised to avoid conception for one month following immunization. Additionally, this vaccine should be administered to all susceptible women preconceptionally.

\section{Varicella}

Preconceptional immunization of women to prevent disease in the offspring, when practical, is preferred to vaccination of pregnant women with certain vaccines. The risks involved for pregnant women who contract varicella include an increased chance of developing severe pneumonia. Risks for the fetus includes congenital varicella (occurs in $2 \%$ of fetuses infected during the second trimester). Live virus vaccine during pregnancy is contraindicated for varicella vaccination, but no adverse outcomes have been reported when given during pregnancy. However, specific immune globulin immunization should be considered for healthy pregnant women exposed to varicella to protect against maternal, not congenital infection. One dose intramuscularly within 96 hours of varicella exposure should be given to the mother.

\section{How preconception care is a strategy to reduce maternal and neonatal/child mortality}

I. Childbirth at an early age is associated with greater health risks for the mother in low- and middle-income countries. Also, complications of pregnancy and childbirth are the leading cause of death in young women aged 1519 years and the adverse effects of adolescent childbearing extend to the health of the infants. Therefore, perinatal deaths are 50\% higher among babies born to mothers under20 years of age than among those born to mothers aged 20-29 years because, babies of adolescent mothers are more likely to be low birth weight, with the risk of long-term effects. However, unintended pregnancies may end in abortions, which are often unsafe resulting in unsafe abortions in women aged 15-19. Proper sex education at early stage of development and adequate child spacing from family planning can help to reduce the occurrence of the risk of the above condition or situations.

II. Untreated STIs can have critical implications for reproductive, maternal and newborn health. Postinfection tubal damage has been implicated in cases of female infertility and women who have had pelvic inflammatory disease are more likely to develop an ectopic pregnancy than those who have not which can result in mortality if they are missed especially when there are no prompt medical interventions. Furthermore, infection with certain types of the human papillomavirus can lead to the development of genital cancers, particularly cervical cancer in women which can be associated with congenital and perinatal infections in neonates, particularly in regions where rates of infection remain high. Also, pregnant women with untreated early infections like syphilis, gonococcal and chlamydia may result in low birth weight, premature deliveries, eye infection stillbirth and neonatal deaths. Therefore, Prevention through prompt and adequate screening to identify health problems and early management of any STIs that are identified are the keys to reducing morbidity and mortality. 34

III. Intimate partner violence refers to behaviour in an intimate relationship that causes physical, sexual or psychological harm, including physical aggression, sexual 
046 Educ. Res.

coercion, psychological abuse and controlling behaviours. Sexual violence is defined as any sexual act, attempt to obtain a sexual act, unwanted sexual comments or advances or otherwise directed against a person's sexuality using coercion, by any person, regardless of their relationship to the person, in any setting, including but not limited to home and work. It includes rape, which is defined as the physically forced or otherwise coerced penetration of the vulvaor anus with a penis, other body part or object.46Intimate partner violence is widespread. A WHO multi-country study found that $15-71 \%$ of women aged $15-49$ years reported experiencing physical and/or sexual violence by an intimate partner at some point in their life. Intimate partner violence and sexual violence directed at women result in a range of health consequences to the women's physical, mental, sexual and reproductive health. Such violence also has negative consequences on the health and well-being of the women's children. Growing up in a family where intimate partner violence happens and being maltreated as a child are associated with violence perpetration later in life, thus contributing to an intergenerational transmission of violence. Intimate partner violence occurring during pregnancy is usually a continuation of ongoing violence, although for some women violence can begin during pregnancy. Health promotion on dating violence, Changing harmful gender norms through comprehensive sexuality education that addresses (gendere quality, human rights, sexuality and sexual relations, community mobilization) empowerment and gender equality and reduction in the harmful use of alcohol can help reduce female violence and the dangers that may occur thereby, reducing the maternal and neonatal/child death.

IV. Mental disorders, in particular depressive and anxiety disorders, in pregnant women and up to 1 year after childbirth pose a serious public health concern because of their adverse effects on infant development, breastfeeding, infant nutritional status and childgrowth.47Studies have documented that a high correlation of antenatal depression may be associated with miscarriage, antepartum haemorrhage, operative delivery, preterm birth and low birth weight.48,49Women with bipolar disorder have a high risk of alcohol and substance use disorders, which could affect fetal outcomes. Management of bipolar disorder in women of childbearing age may be especially challenging because of the potential fetal teratogenic risks of medications. Adequate education and psychosocial counseling for promotion of mental health and psychosocial well-being (for example, enhancement of coping and socio emotional skills, parental training, reduction of psychosocial distress), assessment of psychosocial problems, noting past and ongoing social and relationship issues, living and financial circumstances, and any other ongoing stressful life events will help prevent crises that may disturb the brain and mind thereby, reducing the intake of medications or drugs that can have negative effect on the outcome of pregnancies.

V. The use of alcohol and other psychoactive substances in the preconception period often continues during pregnancy and may impact negatively on the development of the fetus. Alcohol consumption during pregnancy can result in fetal alcohol spectrum disorder, which includes fetal alcohol syndrome, which is often associated with lifelong disability. Continued prenatal exposure to alcohol and drugs can result in neonatal withdrawal syndrome in the newborn. Adequate Screening for substance use, treatment of substance use disorders, including pharmacological and psychological interventions, family planning assistance for families with substance use disorders, including postpartum and between pregnancies and prevention programmes to reduce substance use in adolescents which in turn prevent or reduce the adverse effect of substance use.

VI. Regardless of their pregnancy status, women who smoke tobacco are at increased risk for a wide range of cancers (lung, cervical, pancreatic, bladder, kidney) cardiovascular disease and pulmonary disease.54 Cigarette smoke contains several thousand components and toxins that adversely affect all stages of reproductive function, including folliculo genesis, steroid genesis, embryo transport, endometrial receptivity, endometrial angiogenesis, uterine blood flow and uterine myometrium.55,5Second-hand smoke exposure during pregnancy leads to intrauterine growth retardation and low birth weight, and parental smoking around infants causes respiratory illnesses such as asthma, bronchitis, ear infections and sudden infant death syndrome.64Therefore, cessation of smoking is recommended before pregnancy and proper Screening of women and girls for tobacco use (smoking and smokeless tobacco) at all clinical visits using '5 As' (ask, advise, assess, assist, arrange) is very important. If condition is critical, referral to intensive behavioural counseling services should be done promptly. Female genital mutilation which is a form of identity and continuity, religious perception, sexual control of women, and conformity to socio cultural norms in practicing societies is a form of violence against girls and women. It is a practice that is condoned and perpetuated by certain societies, despite it being a violation of key human rights. Regardless of their pregnancy status, women with female genital mutilation are at increased risk of several health complications, including genital morbidities such as cysts, menstrual and urinal problems, and infections that can affect sexuality and future fertility. Therefore, Preconception Care give room for discussion and discouragement of the practice with the girl and her parents and/or partner, screening of women and girls for female genital mutilation to detect complications that may cause inability to have sexual intercourse, infertility or birth complications, counseling to discourage continuation of female genital mutilation in next 
generation, treatment of infections and other conditions that can cause infertility.

\section{Applied theory}

\section{The health belief model}

The Health Belief Model (HBM) was developed in the early 1950s by social scientists at the U.S. Public Health Service in order to understand the failure of people to adopt disease prevention strategies or screening tests for the early detection of disease. Later uses of HBM were for patients' responses to symptoms and compliance with medical treatments. The HBM suggests that a person's belief in a personal threat of an illness or disease together with a person's belief in the effectiveness of the recommended health behavior or action will predict the likelihood the person will adopt the behavior.

The HBM derives from psychological and behavioral theory with the foundation that the two components of health-related behavior are:

- The desire to avoid illness, or conversely get well if already ill.

- The belief that a specific health action will prevent, or cure, illness.

Ultimately, an individual's course of action often depends on the person's perceptions of the benefits and barriers related to health behavior. There are six constructs of the HBM. The first four constructs were developed as the original tenets of the HBM. The last two were added as research about the HBM evolved.

1. Perceived susceptibility - This refers to a person's subjective perception of the risk of acquiring an illness or disease. There is wide variation in a person's feelings of personal vulnerability to an illness or disease.

2. Perceived severity - This refers to a person's feelings on the seriousness of contracting an illness or disease (or leaving the illness or disease untreated). There is wide variation in a person's feelings of severity, and often a person considers the medical consequences (e.g., death, disability) and social consequences (for example, family life, social relationships) when evaluating the severity.

3. Perceived benefits - This refers to a person's perception of the effectiveness of various actions available to reduce the threat of illness or disease (or to cure illness or disease). The course of action a person takes in preventing (or curing) illness or disease relies on consideration and evaluation of both perceived susceptibility and perceived benefit, such that the person would accept the recommended health action if it was perceived as beneficial.

4. Perceived barriers - This refers to a person's feelings on the obstacles to performing a recommended health action. There is wide variation in a person's feelings of barriers, or impediments, which lead to a cost/benefit analysis. The person weighs the effectiveness of the actions against the perceptions that it may be expensive, dangerous (for example, side effects), unpleasant (for example, painful), time-consuming, or inconvenient.

5. Cue to action - This is the stimulus needed to trigger the decision-making process to accept a recommended health action. These cues can be internal (for example, in the case of maternal health, lower abdominal pain, excessive vomiting and so on) or external (for example, advice from others, illness of family member, newspaper article, etc.).

6. Self-efficacy - This refers to the level of a person's confidence in his or her ability to successfully perform a behavior. This construct was added to the model most recently in mid-1980. Self-efficacy is a construct in many behavioral theories as it directly relates to whether a person performs the desired behavior.

\section{The roles of nurses in preconception care}

According to Anne, Brain and Keith 2014, the essential and critical role of the Nurse in the provision of preconception care is apparent and these may include:

- Health maintenance: This is carried out during antenatal clinics when health education is given and during discharge from one illness or after delivery and follow up visits.

- Family planning: The information starts from when the individual is pregnant. It can be done after six weeks of delivery or anytime of the reproductive year. The information includes the different methods of family planning, advantages and disadvantages.

- To maintain continuity of care (often before, after, and between pregnancies) and close relationships with their patients/ Clients.

It has been reported that women prefer to receive preconception health information from Nurses than because, Nurses are the first contact in any Health Care setting (Fred and Files, 2012). According to Anne et al, 2014, the Nurses can take the following actions to promote preconception health and health care:

- Ask every women of reproductive age whether she intends to become pregnant in the next year. Asking every woman about her reproductive intentions promotes the idea that pregnancies should be intended and planned by providing contraception to the woman who does not intend to conceive and promotes the initiation of preconception care strategies for women if and when they do desire to become pregnant.

- Inform women that health conditions and medications can affect pregnancy outcomes and that pregnancy can affect a woman's health.

- Carrying out outreaches (churches, mosques, market and any other social gathering) to inform the women in the community the importance of taken care of themselves before having sex. 
048 Educ. Res.

\section{SUMMARY}

Preconception Care is the intervention given to male and female of childbearing age to bring a desirable outcome. Maternal age and individual lifestyles can affect outcome of pregnancy, therefore, the group at risk should be identified through timely and proper screening and adequate counseling to give positive pregnancy outcome and optimal reproductive life.

\section{RECOMMENDATION}

The propagation of preconception care should be widely spread in social gathering, religion places (Churches and Mosques) and added to the academic curriculum as early as Junior Secondary School One so that males and females of reproductive age (12-50years) will be well educated from puberty about preconception, since most of the pregnancies among teenagers nowadays are not planned for. Also, the traditional Birth Attendants should be incorporated and well educated on what preconception care is and how to go about it. Nevertheless, Nurses and other health care provider should be well trained and re-trained on preconception care so that they are able to give adequate information to the beneficiaries and in turn, improve their attitude toward having optimal health before getting into relationship that will result into pregnancy. This will therefore reduce the rate of maternal and child mortality. The Federal Government and the other two ties of government should continue sponsor interventions that are in preconception care package so that even the poor in the very remote areas can benefit.

\section{CONCLUSION}

Preconception Care is a vital tool for healthy living, therefore, all males and females of reproductive age and couples should be well guided in their reproductive life to achieve a healthy life. Also, women who have genetic conditions should receive appropriate preconception care and prenatal care, education, and medical counseling to avoid morbidity and mortality for themselves and their future pregnancies.

\section{REFERENCES}

Anne LD, Brain J, Keith F (2014). National Recommendations for Preconception Care: The Essential Role of the Family Physician. The J. the American Board of Family Med. vol. 20 no. 1 81-84

Anne LD, Brain J, Keith F (2014). National Recommendations for Preconception Care: The Essential Role of the Family Physician. The J. the American Board of Family Med. vol. 20 no. 1 81-84.

Boggesss KA, Lieff SL, Murtha AP, Moss K, Beck J, Offenbacer S (2013). Maternal Periodontal Disease is Associated with an Increased Risk for Preeclampsia. Obstetrics Gynaecology, 103: 227-231.
Bourke S, Aitken S, Allen K (2014). National management guidelines for sexually transmissable infections. Sexual Health Society of Victoria J.

Castro LC, Avina RL (2012). Maternal Obestiy and Pregnancy Outcomes. Curr Opin Obstetrics Gynaecology, 14: 601-606.

Centers for Disease Control and Prevention, (2012). Recommendations to improve preconception health and health care in United States. MMWR Recommendations and Reports, 55(06): 119-127.

Centers of Disease Control and Prevention, Sexually Transmitted Diseases, (2012): Treatment Guidelines 2012. Retrieved at: http://www.cdc.gov/std/treatment/2012/specialpops.htm $(11 / 14 / 2012)$.

De Weerd S, Van der AK, Cikot RJ, Braspenning JC (2012). Preconception care: A screening tool for health assessment and risk detection.34:505-511

Ekwempu CC (2014): The influence of antenatal care on pregnancy outcome. Tropical Journal of Obstetrics and Gynaecology 1: 67-71.

Family history of hypertension, heart disease and stroke among women who develop hypertension in pregnancy. American College of Obstetricians and Gynecologists. Obstetrics Gynaecology, 102: 1366-71

Floyd LR, O'Connor MJ, Sokol RJ, Bertrand J, Cordero JF (2015). Recognition and Prevention of Fetal Alcohol Syndrome. Obstetric Gynaecology, 106: 1059-1064.

Good health before pregnancy (2014). preconception care, ACOG Patient Education. American College of Obstetricians and Gynecologists.

Influenza vaccine and treatment during pregnancy (2014). ACOG Committee Opinion No. 305. American College of Obstetricians and Gynecologists, 104: 1125-6.

Jack BW, Atrash H, Coonrod DV (2015): The clinical content of preconception care: An overview and preparation of this supplement. American J. for Obstetric and Gyneacology. (62):66-79.

Johnson K, Samuel FP, Janis B, José FC (2012). Recommendations to Improve Preconception Health and Health Care - United States: A Report of the CDC/ATSDR Preconception Care Work Group and the Select Panel on Preconception Care. Centers for Disease Control and Prevention.

Klerman LV, Jack BW, Coonrod DV (2015): The clinical content of preconception care: Care of psychosocial stressors. American J. for Obstetric and Gyneacology, 199 (62):362-366.

Liu (2010). "Global, regional, and national causes of child mortality: an updated systematic analysis for 2010 with time trends since 2000". The Lancet, 379: 2151-61.

Maternal phenylketonuria (2010). ACOG Committee Opinion No. 230 American College of Obstetricians and Gynecologists.

Naish F, Roberts J (2012). The natural way to better babies. Australia:

Nylander O, Adeyemi O (2010). Antenatal care in developing countries. Baillière's Clinical Obstetrics and Gynaecology, Volume 4, (1): 169186

Obesity in pregnancy (2015). ACOG Committee Opinion No. 315. American College of Obstetricians and Gynecologists. Obstetric Gynecology, 106; 671-5.

Ogle A (2012). Before your pregnancy: Prepare your body for a health

Prenatal and preconceptional carrier screening for genetic diseases in individuals of Eastern European Jewish descent, (2014): American College of Obstetricians and Gynecologists. Obstetric Gynaecology, 104:425-8.

Singh (2010). Unintended Pregnancy: Worldwide Levels, Trends, and Outcomes, 2010. Studies in Family Planning, 41(4): 241-50.

Walsh JA, Feifer CN, Measham AR, Gertler PJ (2013). Maternal and perinatal health problems. Disease Control Priorities in Developing Countries. New York, NY: Oxford University Press.

World Health Organization, World Health Assembly (2013). Draft action plan for the prevention and control of non-communicable diseases 2013-2020. Geneva. 